\title{
La influencia de las mujeres españolas en los resultados de las elecciones generales de 1933
}

\section{The influence of Spanish women in the results of the 1933 General Elections}

\author{
ALEJANDRO CAMINO RODRÍGUEZ \\ Universidad Autónoma de Madrid \\ alejandro.camino@uam.es
}

Resumen: La primera experiencia de las mujeres españolas en unos comicios generales ha recibido escasa atención por parte de los/as historiadores/as profesionales. Normalmente se ha defendido que la orientación mayoritaria de sus votos, al ser consideradas las mujeres españolas como conservadoras y católicas por excelencia, fue un factor fundamental para que se produjese la victoria de las derechas en las elecciones generales de finales de 1933. Este artículo pretende demostrar empíricamente que, aunque las mujeres españolas no hubiesen votado, el giro conservador que tuvo lugar en los resultados de las elecciones de 1933 se hubiese producido de todos modos, debido a la evolución previa del electorado masculino y a la concatenación de factores en la coyuntura que perjudicaban a las opciones políticas de izquierda.

Palabras clave: Elecciones, sufragio universal, género, mujeres españolas, Segunda República.

\begin{abstract}
The first experience of Spanish women in the 1933 general elections has barely received attention from professional historians. Considered as conservative and catholic per excellence, their voting intentions have usually been argued like an essential factor in the victory of the right-wing parties in the general elections occurring at the end of 1933. The aim of this article is to show empirically that even though Spanish women had not voted, the conservative shift the country underwent after these general elections would have happened in any case. This was due to the prior development of the male electorate and the chain of detrimental political events to the left-wing policy options.
\end{abstract}

Keywords: elections, universal suffrage, gender, Spanish women, Second Republic.

Recibido: 1 de noviembre de 2016; aceptado: 3 de enero de 2017; publicado: 21 de septiembre de 2017. Revista Historia Autónoma, 11 (2017), pp. 179-197. 
Introducción

Las elecciones generales celebradas el 19 de noviembre y el 3 de diciembre de 1933, en su primera y segunda vuelta, respectivamente, fueron las primeras en las que hubo sufragio universal en España. Durante muchas décadas, en los estudios históricos y en la opinión pública se consideró que la victoria conservadora en dichos comicios se debió, en gran medida, a que las mujeres votaron mayoritariamente por las derechas, pese a que esta creencia nunca fue demostrada empíricamente.

El presente trabajo tiene como objeto de estudio estas elecciones generales desde una perspectiva de género, con el objetivo de analizar cómo se comportaron electoralmente las mujeres la primera vez en la que estuvieron convocadas a las urnas en unos comicios generales en España. En este artículo parto de la hipótesis de que fue la concatenación de muchos otros factores, ajenos a las mujeres como grupo específico, lo que provocó el giro conservador en las elecciones generales de 1933 respecto a las de 1931 y que, si las españolas no hubiesen votado, también se hubiese producido. Sin embargo, es complejo demostrarlo porque, pese a los intentos de algunas personalidades políticas y de periodistas ${ }^{1}$, a diferencia de en otros países, como en el caso de Chile, las mujeres españolas no votaron en urnas o con papeletas diferenciadas. En consecuencia, la inexistencia de datos electorales disgregados por sexo ocasiona que sea un tema muy difícil de analizar: es imposible conocer con certeza cómo hubiesen sido los resultados de los comicios si solo hubiesen sufragado las mujeres, lo cual provoca que existan algunas limitaciones en los planteamientos utilizados para estudiar la influencia de las españolas en las elecciones.

\section{Estado de la cuestión}

Son abundantes tanto los trabajos que estudian parcialmente los comicios generales de 1933 como aquellos que abordan el papel de las mujeres en el periodo republicano. Sin embargo, son escasos los estudios que han analizado la participación de las españolas en estas elecciones planteándose problemas historiográficos. Estos son los trabajos históricos analizados críticamente en este apartado.

Antes de que apareciesen las primeras investigaciones de historiadores/as, hay una obra esencial que supone el punto de partida en el estudio de esta problemática: el trabajo realizado

${ }^{1}$ Por ejemplo: Diario de Alicante, 26 de octubre de 1933, p. 1; El Noticiero Gaditano, 18 de noviembre de 1933, p. 1. 
por Clara Campoamor en 1936, en el que defiende que si las derechas se impusieron en las elecciones generales de 1933 no fue debido a que las mujeres españolas votasen por estas opciones políticas en mayor medida que los hombres ${ }^{2}$.

Sin embargo, desde entonces, en los estudios históricos que abordaron estos comicios, al menos hasta finales de los años sesenta del siglo xx, se observa que generalmente se soslayó la participación e influencia de las mujeres en estas elecciones o se reforzó la caracterización conservadora y abstencionista de las españolas. Esto se debió a que autores de todas las tendencias historiográficas construyeron sus argumentos sobre la participación femenina en estos comicios de una manera similar, tendiendo a repetir los argumentos de los partidos políticos y de la prensa sobre los resultados electorales en vez de analizar su influencia (o no) en los resultados sin caer en apriorismos.

El estudio de las elecciones republicanas mediante la denominada sociología electoral fue muy común en la historiografía española desde la década de los sesenta hasta finales del siglo xx. Pero fue desde comienzos de los setenta, sobre todo a partir del trabajo de Javier Tusell (1970), cuando los estudios históricos progresivamente comenzaron a problematizar el análisis de la participación electoral de las mujeres en las elecciones de 1933 y la influencia de sus votos en los resultados ${ }^{3}$. Sin embargo, como la mayoría de trabajos de sociología electoral solían repetir la misma metodología ${ }^{4}$, los múltiples estudios de historia general que se realizaron con esta perspectiva apenas generaron avances en el conocimiento sobre el comportamiento electoral de las españolas, con las excepciones de los estudios provinciales de Mercedes Vilanova (1986) y de Fernando Ayala (2001 y 2002), que desmontaron la vinculación entre el sufragio de las mujeres y la "conservadurización" de los resultados 5 .

De todas formas, pese a la existencia de múltiples trabajos provinciales y locales, solo existen dos monografías a nivel nacional. La primera, a cargo de William Irwin (1991), quien no profundizó en la participación electoral femenina, mientras que la segunda fue realizada por Roberto Villa (2011) desde la corriente historiográfica de la nueva historia política. En esta obra, que es el más completo estudio de historia general a nivel nacional sobre estos comicios, el autor se plantea la hipótesis de si los votos de las mujeres fueron decisivos en los resultados electorales, por lo que es un trabajo importante para el estudio de la problemática que el presente artículo pretende abordar. Además, de este autor han sido casi exclusivamente todos los avances históricos en el estudio de estas elecciones durante el siglo XXI, porque con el declive de la

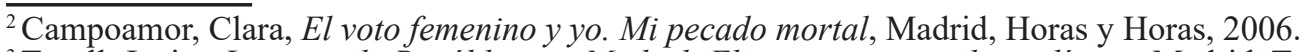

${ }^{3}$ Tusell, Javier, La segunda República en Madrid: Elecciones y partidos políticos, Madrid, Tecnos, 1970.

${ }^{4}$ Tusell, Javier, "El sufragio universal en España (1891-1936): un balance historiográfico", en Ayer, 3 (1991), p. 16.

${ }^{5}$ Vilanova, Mercedes, Atles electoral de la Segona República a Catalunya. Vol. 1: circunmscripcions, comarques i municipis, Barcelona, Enciclopèdia Catalana, SAU, 2005; Ayala, Fernando, Las elecciones en la provincia de Cáceres durante la II República. (1931-1936), Mérida, Editora Regional de Extremadura, 2001; y Ayala, Fernando, "La Segunda República en la provincia de Cáceres. Elecciones y partidos políticos", en XXX Coloquios Históricos de Extremadura, Trujillo, CIT de Trujillo, 2002, pp. 19-28.
} 
sociología electoral los especialistas en procesos electorales mayoritariamente se han centrado en aquellos posteriores al franquismo ${ }^{6}$.

En cuanto al estudio de la participación de las españolas en estas elecciones en las obras de historia de las mujeres y de género, se puede afirmar que comenzó poco después de que algunos trabajos de historia general empezasen tímidamente a problematizar la participación electoral de las mujeres. El primer estudio desde la perspectiva de la historia de las mujeres que se aproximó al tema preguntándose sobre cómo respondieron las españolas ante las elecciones generales de 1933 y de 1936 fue el de Rosa María Capel (1975)7. En esta obra defendió, aunque sin demostraciones empíricas, que las españolas mayoritariamente apoyaron a las derechas o se abstuvieron, pero, gracias a que también analizó la compleja coyuntura electoral, afirmó que el voto femenino no fue el único elemento que provocó el giro conservador ${ }^{8}$. En los años siguientes se publicaron también estudios como los de Geraldine Scanlon (1976) o Concha Fagoaga y Paloma Saavedra (1977), que abordaron parcialmente estas elecciones, aunque sin aportar conclusiones realmente innovadoras respecto al trabajo de Capel. Por tanto, desde la aparición de la historia de las mujeres, se abordaron, aunque no monográficamente, las primeras elecciones ordinarias a Cortes ${ }^{9}$.

Sin embargo, solo la obra de Pablo Villalaín sobre Madrid (2000), que es el estudio fundamental sobre el comportamiento y la participación electoral de las mujeres en noviembrediciembre de 1933 (aunque por su perspectiva provincial debe ser completado), contiene un análisis sistemático del comportamiento electoral de las españolas, gracias a que abordó las elecciones generales republicanas desde una perspectiva de género a través de una metodología que superaba los estudios clásicos de sociología electoral ${ }^{10}$.

Pese al impulso que podría haber supuesto para la historiografía este trabajo, los estudios históricos que abordan las elecciones de 1933 desde una perspectiva de género son todavía prácticamente inexistentes. Por tanto, pese a todos los avances de las últimas décadas, todavía hay muchas lagunas en el estudio de estas elecciones desde una perspectiva de género (algunas de las cuales se pretenden cubrir en este artículo). Este fenómeno se ha producido, por un lado, porque apenas existen trabajos que analicen el comportamiento electoral diferenciado

\footnotetext{
${ }^{6}$ Irwin, William, The 1933 Corts elections: Origin of the Bienio Negro, Nueva York y Londres, Gerland Publishing, 1991; y Villa García, Roberto, La República en las urnas. El despertar de la democracia en España, Madrid, Marcial Pons, 2011.

${ }^{7}$ Capel, Rosa María, El sufragio femenino en la $2^{a}$ república española, Granada, Universidad de Granada, 1975.

${ }^{8}$ El mito del abstencionismo femenino también persiste en muchos estudios recientes. Un ejemplo es: Gómez Cuesta, Cristina, "Mujeres y leyes. Las estudiantes de derecho en la Segunda República", en Folguera, Pilar et al. (eds.), Pensar con la Historia desde el siglo XXI. XII Congreso de la Asociación de Historia Contemporánea, Madrid, UAM Ediciones, 2015, p. 932. Pese a que anteriormente se había desmontado el mismo: Vilanova, Mercedes, Atles electoral... op. cit.

${ }^{9}$ Fagoaga, Concha y Paloma Saavedra, La española ante las urnas, Madrid, Pecosa Editorial, 1977; Scanlon, Geraldine, La polémica feminista en la España contemporánea (1868-1974), Madrid, Siglo XXI, 1976, p. 280. Para un análisis detallado de la evolución historiográfica hasta 1990: Nash, Mary, "Dos décadas de historia de las mujeres en España: una reconsideración”, en Historia Social, 9 (1991), pp. 137-161.

${ }^{10}$ Villalaín, Pablo, Mujer y política, la participación de la mujer en las elecciones generales celebradas en Madrid durante la II república (1931-1936), Madrid, Instituto de la Mujer, 2000.
} 
por sexos. Por otro lado, debido a que no hay ninguna monografía que aborde las elecciones de 1933 desde esta perspectiva para el conjunto del país, lo cual solo será posible cuando existan suficientes trabajos locales y regionales. Por último, esta problemática apenas se ha trabajado desde la renovación interpretativa, metodológica y conceptual que se está produciendo dentro de la historia de género, que ha incorporado desde los años noventa progresivamente una metodología y una perspectiva social, cultural y relacional ${ }^{11}$.

Para terminar con el estado de la cuestión, es importante resaltar que, a pesar de todos los avances historiográficos de las últimas décadas en el análisis de la participación e influencia electoral de las mujeres en estos comicios, muchos autores continúan omitiendo su participación y perviven los trabajos en los que se realizan afirmaciones impregnadas de mitos y apriorismos, lo cual contribuye al mantenimiento de un tópico que ha ido deconstruyéndose gracias a los avances en los estudios históricos ${ }^{12}$. De todas formas, hay que destacar que en la mayoría de los trabajos actuales en los que se mencionan de forma superficial estas elecciones, se expone la participación electoral de las mujeres (y su influencia en los resultados) de una manera acorde a las conclusiones de las investigaciones más destacadas sobre el tema.

\section{Contexto histórico de las elecciones generales de 1933}

En este apartado se hace una breve mención a los antecedentes de la participación política formal de las españolas previa a noviembre de 1933 y se muestra el marco complejo en el que se celebraron estos comicios.

Si bien el voto de las mujeres en unas elecciones generales era algo inédito, las españolas tenían una pequeña práctica política (que no electoral) previa, principalmente en el ámbito municipal. Esto se debió, especialmente, a que durante la dictadura primorriversista se produjo el primer intento estatal de incluirlas en la política formal mediante una legislación limitada de

\footnotetext{
${ }^{11}$ Para la evolución y los debates recientes, por ejemplo: Aguado, Ana, "La historia de las mujeres y del género", en Ortega López, Teresa María (coord.), Por una historia global: El debate historiográfico en los últimos tiempos, Granada, Universidad de Granada y Prensas Universitarias de Zaragoza, 2007, pp. 111-134; o Díaz Sánchez, Pilar, "Historia social e Historia cultural de las mujeres. Apuntes para un debate", en Revista de historiografía, 22 (2015), pp. 13-23.

${ }_{12}$ Esto ocurre incluso en trabajos de algunos de los más importantes y prestigiosos historiadores. Por ejemplo: Townson, Nigel, “«Una República para todos los españoles»: el Partido Radical en el poder, 1933-1935”, en Townson, Nigel (ed.), El republicanismo en España (1830-1977), Madrid, Alianza, 1994, p. 194; Townson, Nigel, La República que no pudo ser. La política de centro en España (1931-1936), Madrid, Taurus, 2002, p. 235; Gil Pecharromán, Julio, La Segunda República, Madrid, Biblioteca Historia 16, 1989, p. 148; López Villaverde, Ángel Luis, Cuenca durante la II República: elecciones, partidos y vida política, 1931-1936, Cuenca, Diputación Provincial de Cuenca, 1997, p. 253; Rodríguez Ranz, José Antonio, Guipúzcoa y San Sebastián en las elecciones de la II República, San Sebastián, Instituto Dr. Camino de Historia Donostiarra, 1994, pp. 424-426 y 495-497; Sancho Calatrava, José Antonio, Elecciones en la II República. Ciudad Real, 1931-1936, Ciudad Real, Biblioteca de autores y temas manchegos, 1989, pp. 208 y 217; y Graham, Helen, "Mujeres y cambio social en la España de los años treinta", en Historia del presente, 12 (2003), p. 16.
} 
derechos políticos, llegando a otorgarse en el Estatuto Municipal de 1924 el voto activo y pasivo a algunas mujeres (las no casadas), aunque nunca llegaron a participar en unas elecciones ${ }^{13}$.

Sin embargo, con la caída del régimen dictatorial las mujeres fueron eliminadas del censo electoral, por lo que no pudieron votar en las elecciones municipales de abril de $1931 \mathrm{ni}$ tampoco en las elecciones constituyentes de ese mismo año, aunque en estas últimas pudieron ser elegibles ${ }^{14}$. Finalmente, en un contexto en el que se reconocieron muchos derechos a las mujeres y en el que constitucionalmente se reformuló la concepción de ciudadanía políticosocial fundamentándola en el principio de igualdad legal de derechos entre ambos sexos, tras muchos debates y obstáculos, el 1 de octubre de 1931 fue aprobado en las Cortes el voto de las mujeres en igualdad con los hombres y ya no hubo en el periodo republicano vuelta atrás. Se inauguraba así una coyuntura más favorable para las españolas, que permitió que tuviesen mayor presencia en la vida pública y política y que posibilitó que hubiese cambios en la vida cotidiana y en las relaciones de género, aunque en la práctica estos fueron escasos.

En cuanto al contexto en el que se celebraron los comicios, hay que tener en cuenta que, aunque en las elecciones constituyentes celebradas el 28 de junio de 1931 la Conjunción Republicano-Socialista obtuvo una amplia mayoría, la correlación de fuerzas en 1933 era muy diferente, como prueba que fuesen adversas para las fuerzas gubernamentales tanto las elecciones municipales parciales de abril de 1933 (cuando por primera vez en España las mujeres sufragaron en igualdad de condiciones) como las elecciones, en septiembre, de vocales para el Tribunal de Garantías Constitucionales. Aunque en ninguna de las dos ocasiones los resultados eran representativos de la sociedad en su conjunto, quedó patente que las derechas habían recuperado posiciones en sus feudos tradicionales y que su capacidad movilizadora tenía muchos beneficios ${ }^{15}$.

Finalmente, el 19 de noviembre se produjo un giro conservador en los resultados electorales que provocó que los partidos que habían gobernado durante la primera legislatura se hundiesen tanto en número de votos como de escaños. La victoria de las derechas es innegable, aunque estuvo maximizada por los efectos de la ley electoral, que establecía un sistema entre mayoritario y mixto que favorecía a las grandes coaliciones y el desequilibrio en el reparto de escaños entre vencedores y vencidos ${ }^{16}$ [fig. 1]. En vista de los datos electorales, no parecen

\footnotetext{
${ }^{13}$ Gómez-Ferrer, Guadalupe y Marta del Moral, "Las pioneras en la gestión local: Concejalas y alcaldesas designadas durante la dictadura de Primo de Rivera y el gobierno Berenguer (1924-1930)", en Nielfa, Gloria (coord.), Mujeres en los Gobiernos locales. Alcaldesas y concejalas en la España contemporánea, Madrid, Biblioteca Nueva, 2015, pp. 41-72.

${ }_{14}^{14}$ Gaceta de Madrid, 10 de mayo de 1931, p. 640.

${ }^{15}$ Sobre las elecciones municipales desde una perspectiva de género: García-Sanz Marcotegui, Ángel, El voto femenino y las elecciones municipales de 1933 en Navarra, Pamplona, Universidad Pública de Navarra, 2009.

${ }^{16}$ La Ley electoral en Gaceta de Madrid, 28 de julio de 1933, p. 635. Salvo que se indique lo contrario, a lo largo del artículo los datos electorales de 1933 están extraídos de Villa García, Roberto, La República...op. cit.; y los de 1931 de Tusell, Javier, Las Constituyentes de 1931: Unas elecciones de transición, Madrid, Centro de Investigaciones Sociológicas, 1982, pp. 161-196.
} 
tener fundamento las tesis que niegan la victoria de las derechas y, como consecuencia, la influencia del voto femenino en los resultados ${ }^{17}$.

Figura 1: Sufragios y escaños alcanzados en la primera vuelta de las elecciones por cada ámbito ideológico

\begin{tabular}{|l|c|c|c|}
\hline \multicolumn{1}{|c|}{ Ámbito ideológico } & Total de votos* & Porcentaje de votos & Escaños \\
\hline Total de las izquierdas & 2804695 & $33 \%$ & 59 \\
\hline Total del centro & 1647271 & $19,5 \%$ & 112 \\
\hline Total de las derechas & 4028749 & $47,5 \%$ & 206 \\
\hline
\end{tabular}

* Suma de las medias de votos que obtuvieron todas las candidaturas de cada opción ideológica en todas las circunscripciones.

Fuente: Elaboración propia a partir de los datos ofrecidos por Villa García, Roberto, La República ... op. cit., pp. 348-351.

Este giro conservador en los resultados estuvo influido por la concatenación de múltiples factores que parecen suficientes, por sí mismos, para impedir la continuidad de cualquier gobierno: el desgaste de los partidos del gobierno, que no satisficieron las expectativas de la mayoría de los sectores, la reorganización y el ascenso de las derechas después de haberse visto desarticuladas en 1931, la ausencia de una coalición electoral entre las izquierdas obreras y los republicanos o de los republicanos entre sí, el abstencionismo que promovió la CNT, la posición mucho más conservadora del Partido Republicano Radical (PRR) respecto a 1931 y la crisis económica global que afectó mucho a España generando fuertes tensiones sociales y laborales. Sin olvidar, por último, que a todos estos factores hay que sumar que la Confederación Española de Derechas Autónomas (CEDA) sacó mucho provecho del despliegue propagandístico y activista sin precedentes que se generó durante la campaña electoral y de la implicación de las asociaciones femeninas en la misma, ya que las derechas, especialmente gracias a Acción Católica de la Mujer (ACM), eran las fuerzas políticas mejor organizadas entre las mujeres.

\section{Ante la primera experiencia electoral de las españolas}

En este apartado se busca retratar, resumidamente, la manera en la que las mujeres se insertaron en la vida política durante la campaña electoral, ya que como era la primera en la que

\footnotetext{
${ }_{17}^{17}$ Por ejemplo: Alejandre García, Juan Antonio, "El voto en España. El Sufragio universal”, en Cuadernos de Historia 16, 126 (1988), p. 32.
} 
los partidos debían atraerse el voto de las españolas, y encima la orientación de sus sufragios era una incógnita, las mujeres ocuparon un lugar destacado en la propaganda y en los discursos. Primero se hace una breve mención a las previsiones de los líderes políticos más destacados sobre la orientación del voto de las españolas, después se repasa la forma en la que las fuerzas políticas más relevantes buscaron atraer sus votos $\mathrm{y}$, para acabar, se exponen unas breves indicaciones de carácter general sobre cómo las mujeres afrontaron esta novedosa situación.

En lo que respecta a las previsiones de los líderes políticos más destacados sobre la orientación del voto de las mujeres, estas fueron variadas, pero mayoritariamente seguían los mismos argumentos que habían mantenido en los debates parlamentarios de 1931.

Dentro del socialismo, las opiniones fueron diversas. Para Indalecio Prieto los votos de las mujeres, quienes no se abstendrían mayoritariamente, perjudicarían especialmente a los partidos republicanos y estarían ligados a los sufragios de los familiares en el caso de las familias socialistas y conservadoras. Sin embargo, tras las elecciones defendió que aunque tuvo influencia, el voto de las mujeres no fue un factor clave que explicase el resultado electoral, lo cual difería del pensamiento de otro socialista destacado como Luis Jiménez de Asúa ${ }^{18}$. Mientras, antes de los comicios, Julián Besteiro, Francisco Largo Caballero y Julián Zugazagoitia confiaban en que los votos de las mujeres favorecerían al socialismo y que, en el peor de los casos, no les perjudicarían en mayor medida que a las derechas ${ }^{19}$.

En el ámbito republicano también hubo diversidad de opiniones. Alejandro Lerroux, el líder del PRR, un partido que se había opuesto casi íntegramente al voto femenino, defendió que las mujeres no iban a alterar sustancialmente los resultados al votar sobre todo hacia unas izquierdas o unas derechas moderadas. Sin embargo, tras las elecciones, cambió radicalmente de discurso y consideró que las mujeres fueron claves para la victoria de las derechas por su religiosidad y por considerarlas incapaces de equilibrar todavía el sentimiento con el entendimiento al tener una escasa preparación política ${ }^{20}$. Mientras, Manuel Rico Avello y Diego Martínez Barrio defendieron que las mujeres votarían lo mismo que sus familiares, por lo que prácticamente se duplicarían los apoyos de todas las candidaturas. Sin embargo, tras las elecciones defendieron que las españolas provocaron la victoria de las derechas, principalmente debido a su catolicismo y a su radicalismo, aunque consideraron que también influyeron otros factores, como el que la desunión de los republicanos fuese muy penalizada por la ley electoral ${ }^{21}$.

\footnotetext{
${ }^{18}$ Heraldo de Madrid, 16 de octubre de 1933, p. 1; La Vanguardia, 17 de octubre de 1933, p. 28; Las Provincias, 28 de noviembre de 1933, p. 10. Jiménez de Asúa, Luis, La constitución de la democracia española y el problema regional, Buenos Aires, Losada, 1946, pp. 34-35.

${ }^{19}$ Heraldo de Madrid, 4 de noviembre de 1933, p. 8; Heraldo de Madrid, 8 de noviembre de 1933, p. 11; La Voz de Asturias, 9 de noviembre de 1933, p. 1; El Sol, 18 de noviembre de 1933, pp. 1-2; La Voz, 18 de noviembre de 1933, p. 4.

${ }^{20}$ Heraldo de Madrid, 1 de noviembre de 1933, p. 13; Heraldo de Madrid, 8 de noviembre de 1933, p. 1; La Libertad, 18 de noviembre de 1933, p. 1; El Sol, 18 de noviembre de 1933, p. 1; La Voz, 18 de noviembre de 1933, p. 4; El Sol, 21 de noviembre de 1933, p. 1; $A B C, 21$ de noviembre de 1933, p. 55.

${ }_{21}^{2}$ Heraldo de Madrid, 30 de octubre de 1933, p. 8; Heraldo de Madrid, 11 de noviembre de 1933, p. 16; La Libertad, 7 de diciembre de 1933, p. 5; Martínez Barrio, Diego, Memorias, Barcelona, Planeta, 1983, pp. 84-85.
} 
Sin embargo, a diferencia de los líderes del PRR, los republicanos de izquierda apenas se manifestaron en la campaña electoral sobre esta incertidumbre. Mientras personas como Manuel Azaña, antes de los comicios, consideraban que los partidos conservadores se verían beneficiados al estar influidas las mujeres por la religión, otras personas fueron todavía más lejos al defender que estas elecciones demostrarían si las españolas se merecían (o no) la emancipación y la igualdad legal respecto a los varones, en función de donde depositasen mayoritariamente sus votos ${ }^{22}$. Sin embargo, tras las elecciones hubo diversidad de opiniones entre quienes, como Marcelino Domingo, consideraban que las mujeres no habían tenido ningún papel determinante en el giro conservador y entre los que defendieron que fueron decisivas al votar sin la cultura política necesaria y estando totalmente influidas por la religión ${ }^{23}$.

Respecto al ámbito de las derechas, sus líderes, que habían defendido el reconocimiento del sufragio femenino confiados en su propio beneficio electoral, normalmente interpretaron que, por el hecho de ser mujeres, las votantes eran mayoritariamente católicas y conservadoras. Sin embargo, el líder de la CEDA, José María Gil Robles, antes de las elecciones manifestó que no consideraba que fuesen a ser determinantes en una hipotética victoria de su partido ${ }^{24}$.

Como se aprecia al analizar las opiniones de algunos líderes de las diferentes opciones políticas, todas las previsiones fueron maniqueas, simples, interpretaban a las mujeres como un todo homogéneo y reforzaban la idea de que la política era un ámbito ajeno a ellas. Ello provocó que muchos cambiasen sus discursos tras el día 19, o bien para culparlas o bien para exaltarlas, en función de sus propios resultados electorales. Por tanto, a pesar de que las españolas vieron reconocidos sus derechos democráticos y la igualdad jurídica, la igualdad social entre sexos todavía estaba lejos.

Respecto a la propaganda que buscó captar el voto de las mujeres, los partidos políticos más importantes fundamentalmente dirigieron sus mensajes a las españolas aludiéndolas en su teórica condición de madres, esposas e hijas y, además, trataron de atraer sus sufragios utilizando argumentos emocionales y haciendo referencia a las supuestas cualidades naturales que poseían diferentes a las de los varones. Por tanto, ante estos estereotipos compartidos, las distintas fuerzas utilizaron argumentos similares pero interpretados desde ópticas diferentes. Esta estrategia se debió a que pervivían en el imaginario de todas las culturas políticas muchos rasgos de los modelos de género tradicionales y a que esto no era contradictorio con el ideal de género que los dirigentes republicanos buscaron implantar, ya que en el mismo, mientras se defendía la igualdad legal entre sexos, también se establecía la idea de la diferenciación sexual, por la cual las mujeres tenían unas funciones particulares, principalmente la maternidad ${ }^{25}$.

\footnotetext{
$\overline{{ }^{22} \text { El Luchador, } 4}$ de noviembre de 1933, p. 1; Heraldo de Madrid, 4 de noviembre, p. 8; ABC, 16 de noviembre de 1933, p. 23; La Libertad, 16 de noviembre de 1933, p. 3; La Vanguardia, 16 de noviembre de 1933, p. 23.

${ }^{23}$ Las Provincias, 26 de noviembre de 1933, p. 9.

${ }^{24} A B C, 20$ de octubre de 1933, p. 22.

${ }^{25}$ Un desarrollo de estas ideas en Aresti, Nerea, Médicos, Donjuanes y Mujeres Modernas. Los ideales de feminidad y masculinidad en el primer tercio del siglo XX, Guipúzcoa, Editorial de la Universidad del País Vasco, 2001.
} 
Las peculiaridades más destacadas fueron que mientras la CEDA buscó atraerse los votos femeninos resaltando principalmente que debían defender, en mayor medida incluso que los hombres, lo que consideraban los valores tradicionales de la sociedad española (orden, religión católica, familia, patria y propiedad), la mayoría de partidos republicanos y el Partido Socialista Obrero Español (PSOE) buscaron obtener réditos electorales de que durante el primer bienio republicano se hubiese conseguido una igualdad formal de derechos entre ambos sexos, independientemente de que lo hubiesen apoyado o no.

Sin embargo, las mujeres no solo fueron receptoras pasivas de la propaganda electoral: aunque las militantes normalmente tuvieron que subordinarse a las directrices políticas y estratégicas de los diferentes partidos, cuyos órganos de dirección, salvo excepciones, estaban copados íntegramente por hombres, colaboraron en buena parte de las funciones propagandísticas de las diversas opciones políticas. Esta labor fue especialmente importante en la CEDA y en el PSOE, ya que desde el reconocimiento del sufragio de las mujeres buscaron movilizar a las españolas y conseguir sus votos. Sin embargo, los partidos del ámbito republicano no hicieron en conjunto grandes esfuerzos por atraer a las mujeres a sus filas ni por competir con fuerza por sus votos, ya que mayoritariamente las identificaban como contrarias a sus ideales al ser teóricamente conservadoras y estar influidas por la Iglesia.

A rasgos muy generales, se puede afirmar que la participación de las conservadoras se restringió en su mayoría a labores de menor visibilidad, auxiliares y sociales, con las importantes excepciones de mujeres destacadas como la cedista Francisca Bohigas o la tradicionalista María Rosa Urraca Pastor. Además, para los líderes conservadores la incorporación de las mujeres a la esfera política y pública se consideraba coyuntural, debiendo regresar tras las elecciones a lo que consideraban sus ocupaciones tradicionales y su verdadera función social ${ }^{26}$. Mientras, la gran diferencia de las mujeres socialistas respecto a las conservadoras fue que estas tuvieron un mayor protagonismo y presencia pública durante la campaña, de hecho, las militantes más destacadas participaron en mítines en mayor medida que las conservadoras y las republicanas más relevantes. También las mujeres participaron en la campaña electoral a través de las organizaciones femeninas, las cuales, pese a que solían tener una escasa penetración entre las españolas, trataron de fomentar la participación de las mujeres en la contienda electoral buscando, normalmente, recabar el voto para las fuerzas políticas más afines a sus posicionamientos ideológicos ${ }^{27}$.

Sobre lo analizado se puede afirmar que todas las fuerzas siguieron identificando a las mujeres, en mayor o menor medida, como portadoras de características propiamente femeninas diferentes a las de los varones y con el estereotipo de religiosa, pese a que la identidad católica era una entre muchas y no necesariamente la predominante. Esto se debe a que la igualdad legal

\footnotetext{
${ }^{26}$ Esta idea también se aprecia en Urraca Pastor: La Constancia, 15 de noviembre de 1933, p. 2; pero no en otras líderes como Bohigas: Camino Rodríguez, Alejandro, "Francisca Bohigas: mujer católica, diputada y pedagoga (1928-1950)", en Ibero-Americana Pragensia, 46 (2017), en prensa.

${ }^{27}$ Para ampliar la información: Villalaín, Pablo, Mujer y política ... op. cit., pp. 184-228.
} 
no supuso cambios inmediatos en las mentalidades de los españoles, pese a que las relaciones de género estaban transformándose (muy lentamente) en la mayoría de ámbitos políticos y sociales.

Además, deseo destacar que, aunque los varones habían disfrutado del sufragio universal masculino desde 1890 y tenían de media un mayor nivel educativo que las mujeres (aunque las tasas de analfabetismo entre las españolas en 1933 eran relativamente similares a las de los varones en 1890$)^{28}$, la mayoría de hombres estaban tan (o tan poco) politizados como las mujeres y tenían tanta (o tan poca) formación política como las españolas. Esto provocó que las mujeres, especialmente en ciertos sectores politizados, tuviesen capacidad suficiente como para decidir por sí solas su sufragio, aunque fuese a las derechas, y para participar de las corrientes mayoritarias de voto en cada circunscripción. De todas formas, no es descabellado pensar que muchas votasen por las mismas opciones políticas que sus familiares, ya que las mujeres y los hombres son personas que, por autónomas que sean, están influidas por la pertenencia a un entorno concreto. Esto provoca que sea lógico que los miembros de un mismo núcleo familiar sufraguen por opciones ideológicas próximas.

También es necesario resaltar que la presencia de las mujeres siguió siendo muy escasa en las candidaturas: a pesar de que se multiplicase por cuatro el número de candidatas respecto a 1931, el aumento vino dado sobre todo por el mayor número de listas electorales que concurrieron a los comicios. Además, como la mayoría de ellas tenían escasas opciones de ser elegidas, el número de diputadas (aumentaron de tres a cinco respecto a 1931) siguió siendo cuantitativamente marginal y no proporcional a la participación política femenina, lo cual fue una situación que recibió muchas críticas, también entre las mujeres conservadoras ${ }^{29}$.

Por tanto, el reconocimiento del derecho a voto a las mujeres no supuso ni su plena integración en la vida política oficial ni la consecución de la igualdad real, aunque fue un punto de partida que no pudo desarrollarse por la llegada del franquismo. Sin embargo, pese las limitaciones, la participación de las mujeres en la política se incrementó de forma importante: aumentó su afiliación a los partidos y sindicatos, creció su protagonismo en los discursos y programas de los mismos, se multiplicó el número de propagandistas y candidatas, etc. Además, la reapropiación por parte de las mujeres de los discursos de sus partidos políticos preferidos les permitió acceder a las ventajas de la igualdad y de la libertad y, redefinir, desde su propia identidad y experiencia, un nuevo orden simbólico y autorreferencial para articular desde ellos sus intereses, dar significado a sus acciones y construir su identidad como agentes sociales ${ }^{30}$. En definitiva, la primera experiencia electoral para las españolas fue algo importante porque

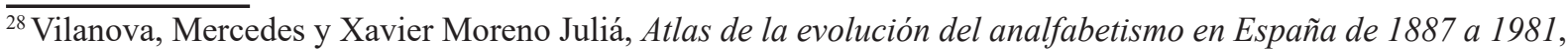
Madrid, Ministerio de Educación, Cultura y Deporte, 1992, pp. 413 y 429.

${ }^{29}$ Por ejemplo: Acción Católica de la Mujer, 5 de noviembre de 1933, p. 5; Ellas, 10 de diciembre de 1933, p. 1; Acción, 22 de diciembre de 1933, p. 1.

${ }^{30}$ Aguado, Ana, "Identidades de género y culturas políticas en la Segunda República", en Pasado y Memoria. Revista de Historia Contemporánea, 7 (2008), p. 124.
} 
muchas de ellas asumieron papeles que nunca habían desempeñado en la esfera pública y política, lo cual contribuyó a la lenta evolución de los modelos de género hegemónicos que venía produciéndose durante el primer tercio del siglo $\mathrm{xx}^{31}$.

\section{El reflejo en la prensa de la participación electoral de las mujeres}

En este apartado se examinan los análisis que hicieron los periódicos sobre la posible influencia de los votos de las mujeres en los resultados electorales, antes y después de la celebración, el 19 de noviembre, de la primera ronda electoral, ya que la mayoría de los medios se posicionaron sobre este tema.

En las semanas anteriores a la celebración de las elecciones, la mayoría de los diarios monárquicos y/o próximos a las derechas, de forma acorde a la actuación de sus partidos políticos afines, mantuvieron una propaganda que apelaba al lado sentimental de las mujeres y las interpelaron constantemente aludiendo a la utilidad de sus votos para salvar tanto la unidad de España como la religión católica ${ }^{32}$. Además, la mayoría de estos medios defendieron antes de los comicios que ganarían las derechas gracias a los votos de las mujeres, al estar teóricamente influidas por el catolicismo y por tener las españolas un claro carácter conservador ${ }^{33}$.

También los medios afines a las izquierdas obreras y a los republicanos usaron los argumentos que más frecuentemente utilizaron los partidos a los que apoyaban. Por ejemplo, Heraldo de Madrid, cercano a los republicanos de izquierda, pidió el voto dirigiéndose a las españolas en su condición de esposas o madres y reivindicando que debían apoyar a los partidos republicanos porque les habían reconocido los derechos políticos y sociales que ponían los pilares básicos para su emancipación ${ }^{34}$.

Todos los periódicos del día posterior a la celebración de las elecciones resaltaron el carácter masivo de la participación de las mujeres, no solo en calidad de electoras, y la importancia que le dieron al ejercicio del derecho que por primera vez disfrutaban. Sin embargo, en el debate postelectoral, como todos buscaron responsables inmediatos del giro conservador en los resultados, fueron muchos quienes lo achacaron, bien como la única causa o bien como una de las principales, a las mujeres en general, y a Clara Campoamor, la gran defensora parlamentaria del reconocimiento del sufragio femenino, en particular. Esto se debió a que mayoritariamente

\footnotetext{
${ }^{31}$ Para las lentas transformaciones en los modelos de género: Blasco, Inmaculada, Paradojas de la ortodoxia. Política de masas y militancia católica femenina en España (1919-1939), Zaragoza, Prensas Universitarias de Zaragoza, 2003; Aresti, Nerea, Masculinidades en tela de juicio, Madrid, Cátedra, 2010.

${ }^{32}$ Por ejemplo: $A B C, 14$ de noviembre de 1933, p. 29.

${ }^{33} A B C, 24$ de octubre de 1933, p. 3; El Siglo futuro, 11 de noviembre de 1933, p. 1; $A B C, 17$ de noviembre de 1933, p. 3; $A B C, 1$ de diciembre de 1933, p. 15 .

${ }^{34}$ Heraldo de Madrid, 6 de noviembre de 1933, p. 1; Heraldo de Madrid, 14 de noviembre de 1933, p. 7.
} 
se interpretó que la orientación política de las mujeres era mucho más conservadora que la de los hombres, incluso donde ganaron las izquierdas.

Para los medios conservadores, las mujeres decidieron en buena parte los resultados electorales y tuvieron un comportamiento ejemplar al confirmar que eran mayoritariamente conservadoras, pero su protagonismo en la prensa afín fue menor del que podía esperarse después del papel casi decisivo que les concedieron en la propaganda electoral. No hubo apenas artículos que valorasen específicamente su actuación más allá de los que reclamaban que debían volver a sufragar en la segunda vuelta en el mismo sentido ${ }^{35}$.

Asimismo, fueron también muchos los medios republicanos, progresistas y de izquierdas los que tras las elecciones defendieron que las derechas vencieron debido a los votos de las mujeres, que estaban totalmente influidos por su religiosidad. Heraldo de Madrid fue uno de los medios más tajantes en este sentido, defendiendo que las mujeres habían renegado de los derechos que el régimen les había concedido y llegando a plantear que la concesión de estos derechos fue precipitada ${ }^{36}$.

Ante los agravios que las mujeres españolas estaban sufriendo, Clara Campoamor en diversas ocasiones se vio en la necesidad de justificar y reivindicar su actuación parlamentaria, los derechos conseguidos por las mujeres y que el voto femenino no había provocado la derrota de los partidos republicanos, señalando otros factores (ausentes en la mayoría de los análisis) y buscando demostrarlo empíricamente comparando los resultados de 1933 con los de $1931^{37}$.

De todas formas, también hubo analistas y medios de comunicación que sostuvieron que los votos de las mujeres no eran, ni mucho menos, tan importantes en el cambio de orientación de los resultados electorales como muchos defendían, ya que estos sí valoraron otros aspectos que pudieron tener incidencia en el resultado de los comicios. Para estas voces, las mujeres eran solo una cabeza de turco debido a que la opinión pública no estaba fragmentada en función del sexo y a que el voto de los varones no se mantuvo necesariamente estable respecto a $1931^{38}$. Además, hubo políticos y periódicos que, al ser conscientes de que era casi imposible que ninguna fuerza de izquierdas garantizase mayoritariamente la fidelidad del electorado que les votó en 1931, predijeron antes de los comicios aproximadamente el resultado electoral, por lo que los resultados no fueron sorprendentes para quienes tuvieron en cuenta la compleja coyuntura en la que se celebraron las elecciones ${ }^{39}$.

\footnotetext{
${ }_{35}$ ABC, 21 de noviembre de 1933, p. 23; La Nación, 21 de noviembre de 1933, p. 1; El Diario Palentino, 23 de noviembre de 1933, p. 2; ABC, 24 de noviembre, p. 3; La Nación, 2 de diciembre de 1933, pp. 3-5.

${ }^{36}$ Heraldo de Madrid, 23 de noviembre de 1933, p. 1; Heraldo de Madrid, 24 de noviembre de 1933, p. 16. Para otros ejemplos: La Libertad, 7 de diciembre de 1933, p. 5; La Voz de Menorca, 14 de diciembre de 1933, p. 1; Diario de Almería, 15 de diciembre de 1933, p. 1.

${ }^{37}$ Heraldo de Madrid, 19 de octubre de 1933, p. 16; Heraldo de Madrid, 25 de noviembre de 1933, p. 16; Luz, 25 de noviembre de 1933, p. 12; Las Provincias, 26 de noviembre de 1933, p. 9; La Libertad, 29 de noviembre de 1933 , p. 5.

${ }^{38}$ El Luchador, 21 de noviembre de 1933, p. 1; La prensa, 26 de noviembre de 1933, p. 1; Diario de Almería, 30 de noviembre de 1933, p. 1; La Libertad, 15 de diciembre de 1933, pp. 3-4.

${ }^{39} A B C, 17$ de octubre de 1933, p. 21; $A B C, 16$ de noviembre de 1933, p. 23; Heraldo de Madrid, 18 de noviembre de 1933 , p. 2 ; $A B C 19$ de noviembre de 1933, p. 43.
} 
De todas formas, lo ocurrido en 1933 no tuvo continuación tras las elecciones generales de 1936, en las que se impuso el Frente Popular: los líderes políticos y la prensa justificaron en menor medida los resultados con la orientación de los votos de las españolas ${ }^{40}$. Esto se debe a que comenzaron a ser conscientes de que no se podía responsabilizar a la mitad del electorado de unos resultados sin analizar la coyuntura en la que se celebraban las elecciones. Si en 1936 fue posible la victoria de las izquierdas se debió a que el contexto general era muy diferente al de finales de 1933, el cual provocó que mujeres y hombres que antes habían apoyado a las derechas entonces sufragasen por las izquierdas.

\section{Estudios de caso sobre los resultados de las elecciones generales de 1933}

En este apartado se analizan los resultados de los comicios en las circunscripciones de Madrid (capital y provincia) y de Málaga (capital) para reivindicar que estos no favorecen la interpretación de que el cambio en la orientación de los resultados electorales de 1933 respecto a 1931 fuese provocado por las españolas. La elección de estas circunscripciones se debe, en el caso madrileño, a la gran importancia y simbolismo que tenía la capital española y a que hubo victorias de diferentes fuerzas políticas, mientras que, en el caso malagueño, obedece a que hubo algunas peculiaridades respecto al resto de España que arrojan luz sobre los resultados globales: hubo mucha incidencia del abstencionismo anarquista y fue donde el Partido Comunista de España (PCE) tuvo más fuerza favorecido por su estrategia pragmática y emocional ${ }^{41}$.

En ambas circunscripciones madrileñas en la primera vuelta de las elecciones se produjo una victoria de los candidatos socialistas por un estrecho margen. En Madrid capital votaron 388969 personas, cuando en total había 294278 electoras y 224204 electores, por lo que sufragó un $75,02 \%$ del electorado. Si se tiene en cuenta que el 28 de junio de 1931 hubo un $67,97 \%$ de votantes sobre el censo electoral (exclusivamente masculino), se puede afirmar que las mujeres acudieron en masa a las urnas y, si se hace la estimación de que ambos sexos sufragaron en la misma proporción, votaron en 1933 en torno a 168200 hombres y 220769 mujeres $^{42}$. De estos hombres, como mínimo, los 26046 que votaron de media a los candidatos de Acción Nacional (AN) en las elecciones constituyentes volverían a votar a las derechas. Entonces, incluso en la hipótesis extrema e imposible de que el resto de hombres en 1933 votasen por las opciones de izquierda, republicana y obrera, nos encontramos con la imposibilidad de que todos los sufragios masculinos hubiesen ido a parar a estos candidatos, porque los 142154 votos

\footnotetext{
${ }^{40}$ Heraldo de Madrid, 20 de febrero de 1936, p. 2.

${ }^{41}$ Un ejemplo representativo del beneficio de esa estrategia en: Campoamor, Clara, El voto... op. cit., p. 245.

${ }^{42}$ Anuario Estadístico 1932-1933, Madrid, Sucesores de Rivadeneyra, 1934 y Anuario Estadístico 1934, Madrid, Sucesores de Rivadeneyra, 1935.
} 
restantes de los varones solo serían suficientes para cubrir la media de sufragios obtenidos por los candidatos socialistas $(140920)^{43}$.

Mientras, en la circunscripción de Madrid provincia votaron 153209 personas $^{44}$, cuando había 105553 electores y 106728 electoras. Como en las elecciones constituyentes sufragó un 71,27\% del censo electoral, si se hace la estimación de que se repitiese el mismo porcentaje de votantes masculinos, sufragaron en esta primera vuelta 75228 hombres $^{45}$. Si a esta cifra se le resta el número de votos que de media obtuvieron los candidatos de AN en las constituyentes (13603), porque probablemente estos hombres seguirían optando por las opciones conservadoras, incluso en el caso extremo de que los 61625 hombres restantes se decantasen por las opciones de izquierdas, en solitario no habrían podido conseguir los votos totales que de media obtuvieron los candidatos de las cuatro candidaturas de izquierdas (68426). Los cálculos de ambas circunscripciones muestran que es erróneo afirmar que el incremento electoral de las derechas viniese propiciado en exclusiva por los votos de las mujeres: los hombres restaron apoyos a las izquierdas y multiplicaron sus apoyos a los partidos conservadores.

Sin embargo, en la segunda vuelta electoral, celebrada solo dos semanas después, el resultado cambió: mientras que en la capital el PSOE obtuvo los escaños de la mayoría tras una victoria ajustada, en la provincia los obtuvieron las derechas. Como no hubo tiempo para que se modificase ideológicamente el sentido del voto de parte del electorado, esto se debió a las diferentes actitudes que tomaron los electores radicales, porque la CEDA y el PSOE concentraron los sufragios de los votantes que claramente eran de derechas o de izquierdas, respectivamente.

A diferencia de en otras circunscripciones, en Madrid no hubo alianzas entre el PRR con las derechas. Por esta razón, el PRR no pidió el voto a sus electores para ninguna fuerza ${ }^{46}$. Entonces, mientras en Madrid capital la candidatura del PSOE pudo hacerse con los escaños de la mayoría gracias a que la lista de la CEDA no consiguió atraer a la mayoría de votantes del PRR, en la circunscripción provincial la candidatura derechista obtuvo una estrechísima victoria porque pudo atraer a buena parte del electorado republicano conservador-moderado. En la provincia, además, Manuel Alonso Zapata, que fue el candidato de izquierdas que más votos obtuvo (69874), consiguió casi mil votos menos que el candidato de la Conjunción Republicano-Socialista más apoyado en 1931, Luis Fernández Clérigo, pese a que se había doblado el censo electoral.

En el estudio de caso de Málaga capital se analiza exclusivamente la segunda vuelta de las elecciones, porque para la misma se formaron dos amplias coaliciones que se disputarían los escaños de las mayorías: una compuesta por los agrarios, la CEDA y el PRR y otra integrada

\footnotetext{
$\overline{{ }^{43} \text { Eliminando del }}$ cálculo a Besteiro porque su prestigio atrajo a votantes que no sufragaron por el resto de su candidatura.

${ }^{44}$ Según el Anuario Estadístico 1934... op. cit. (151789 según el Boletín oficial de la Provincia de Madrid).

${ }^{45}$ Anuario Estadístico 1932-1933... op. cit.

${ }^{46}$ Villalaín, Pablo, Mujer y política... op. cit., p. 275.
} 
por el PCE, el PSOE y los radicales-socialistas, la cual se denominó Frente Único Antifascista. Fue esta última coalición la que obtuvo la victoria.

En total votaron 52269 personas, habiendo 40060 electores y 47667 electoras ${ }^{47}$. Si se hace la estimación de que se repitiese el mismo porcentaje de votantes varones que en las elecciones generales de 1931 (53,24\%), acudieron a las urnas en esta segunda vuelta 21328 hombres. De ellos, los 997 que votaron a las derechas en 1931 probablemente volverían a sufragar en la misma dirección. Por tanto, incluso en la hipótesis imposible de que el resto de hombres sufragasen por el candidato más votado (Aurelio Ramos Acosta, 30,038 votos), al menos 9707 mujeres también lo hicieron. Además, en el caso de la capital malagueña tampoco los diferentes candidatos de izquierdas o republicanos pudieron mantener sus apoyos masculinos de junio de 1931. Pese a que se duplicó el electorado, el socialista Antonio Fernández Bolaños, el radical Pedro Armasa y el radical-socialista Francisco Saval vieron disminuidos sus apoyos en 2904, 4464 y 6697 , respectivamente. Entonces, tanto el crecimiento de las derechas respecto a 1931 como la nueva victoria de las izquierdas (pese a la pérdida de votos) lo provocaron las y los malagueños en conjunto y no solo las mujeres ${ }^{48}$.

Sin embargo, en la circunscripción provincial las derechas coaligadas fueron ampliamente vencedoras porque el PSOE en solitario no pudo hacerles frente, aunque la unión de los partidos de izquierda obrera y burguesa no hubiese sido suficiente para que obtuviesen los escaños de las mayorías. Además, quiero resaltar que sería un error interpretar que la menor fuerza de las izquierdas en la provincia se debía a la acción de las mujeres, porque como demostró un estudio sobre Cáceres, incluso en las zonas rurales donde los sectores conservadores eran hegemónicos las españolas no tuvieron un comportamiento electoral significativamente diferente al de los hombres ${ }^{49}$.

Terminados estos análisis, se expone a continuación una valoración de los resultados electorales en el conjunto de España.

A las mujeres no se les puede atribuir ningún tipo de responsabilidad en que las fuerzas de izquierdas se presentasen separadas electoralmente frente a las coaliciones derechistas, lo que les impidió traducir sus apoyos en escaños. Es cierto que en muchas circunscripciones (como en el caso de Málaga provincia) las izquierdas no hubiesen obtenido mayor rédito parlamentario de una coalición, pero en otras sí. Esto lo demuestran casos como el de la capital malagueña, donde si no hubiesen formado una coalición no habrían obtenido los diputados de las mayorías, o el de la circunscripción de Barcelona capital, donde, tras ser derrotadas las izquierdas ajustadamente en noviembre de 1933, la situación se revirtió solo dos meses después cuando la Coalició d'Esquerres consiguió la mayoría absoluta en las elecciones municipales.

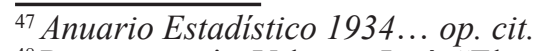

${ }^{48}$ Para otra tesis: Velasco, José, "Elecciones de 1933 en Málaga”, en Jábega, 29 (1980), p. 38.

${ }^{49}$ Ayala, Fernando, Las elecciones ... op. cit., p. 139.
} 
También es importante destacar que si los resultados de estos comicios no mostraron una estabilidad respecto a las elecciones constituyentes se debió a que entonces las opciones políticas conservadoras, como estaban desarticuladas, no vieron traducida en votos su implantación político-social, lo cual sí ocurrió desde las municipales de 1933. Solo desde entonces las distintas opciones ideológicas vieron traducida en apoyos la fuerza social que habían tenido tradicionalmente en cada circunscripción. Por tanto, la cultura política de los diferentes territorios se mantuvo relativamente estable respecto al periodo de la restauración ${ }^{50}$.

En consecuencia, en el gran auge de las derechas en 1933 respecto a 1931 ni mujeres ni hombres por separado tuvieron realmente importancia. Esto se debe a que el comportamiento electoral de las y los españoles estaba guiado, más que por el sexo, por la coyuntura, por su pertenencia a un determinado grupo socioeconómico y por las identidades que en cada persona primasen, ya que la identidad católica o la de clase solo eran unas de las posibles y no necesariamente las predominantes.

Todo esto demuestra que frecuentemente no existió un verdadero esfuerzo de analizar empíricamente los resultados electorales ni el comportamiento electoral de las españolas. Fue más sencillo justificar los resultados con la actitud de las mujeres, pese a que en muchas ocasiones las tesis eran insostenibles. Algunos de los ejemplos más claros son que diversos autores hayan asegurado que las españolas dieron el triunfo a las fuerzas derechistas en Palencia y Álava, pese a que las candidaturas conservadoras obtuvieron, respectivamente, el $70 \%$ y el $81,3 \%$ de los sufragios ${ }^{51}$.

Por último, creo conveniente destacar, por un lado, que las españolas votaron a candidatos en función de su ideología o intereses de carácter general, por lo que no sufragaron de acuerdo con sus identificaciones específicas de género $\mathrm{y}$, por otro lado, que a las candidatas no les perjudicó normalmente de forma clara su condición de mujeres, ya que solían recibir un número de votos acorde al de sus compañeros de candidatura, siendo Francisca Bohigas (que pese a todo consiguió el acta de diputada por León) quién más desfavorecida fue por este motivo.

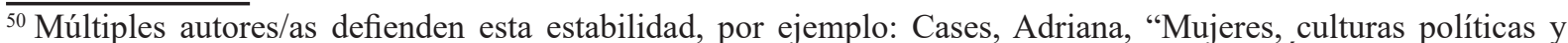
movilización electoral en la Segunda República. Alicante (1931-1936)”, en Barrio Alonso, Ángeles et al. (eds.), Nuevos horizontes del pasado: culturas políticas, identidades y formas de representación, Santander, Ediciones de la Universidad de Cantabria, 2011, pp. 15-16; Vilanova, Mercedes, "La elección legislativa del 19 de noviembre de 1933 en Cataluña y la tergiversación historiográfica del voto de la mujer", en Haciendo historia: homenaje al profesor Carlos Seco, Madrid, Universidad Complutense, 1989, p. 534; García García, Cristóbal, Partidos y elecciones: 1933 en Huelva, Huelva, Universidad de Huelva, 1996, p. 200; Pablo Contreras, Santiago de, La $2^{a}$ República en Álava. Elecciones, partidos y vida política, Leioa, Editorial de la Universidad del País Vasco, 1989, p. 319; Sánchez, José y Miguel Ángel Mateos Rodríguez, Elecciones y partidos en Albacete durante la II República. 1931-1936. Análisis demográfico, actividad económica. Sociología electoral y comportamiento político, Albacete, Tall, 1977, pp. 142-143; Ortega, Miguel Ángel, "Las Elecciones de la Segunda República en Cuenca, el papel del Continuismo", en Espacio, tiempo y forma. Serie V: Historia contemporánea, 1 (1988), pp. 243-260.

${ }^{51}$ Pozo Gutiérrez, Leandro, "Elecciones y partidos políticos en Palencia durante la II República", en Publicaciones de la Institución Tello Téllez de Meneses, 48 (1983), pp. 134 y 152; Cillán-Apalategui, María del Coro, "Sociología electoral de Álava. 1931-1936", en Vasconia: Cuadernos de historia-geografía, 3 (1984), p. 245.
} 


\section{Conclusiones}

La primera experiencia del sufragio universal en España fue muy importante para las mujeres porque supuso un paso más en el proceso de su incorporación al ámbito político, tal y como se había ido configurando en la España liberal. Se ha considerado, tanto en buena parte de los medios de comunicación contemporáneos como en muchos trabajos históricos posteriores, que el voto femenino fue decisivo o muy importante para la victoria de las derechas en las elecciones generales de 1933. Sin embargo, la realidad fue mucho más compleja: el giro conservador de los resultados en relación a los de las elecciones constituyentes de 1931 se debió al cambio en la correlación de fuerzas entre las diferentes opciones políticas, provocado tanto por la concatenación de los factores de la coyuntura española (expuestas en el apartado correspondiente) como por el hecho de que las derechas hubiesen recuperado el dominio en sus zonas de arraigo tradicional. Entonces, tanto por la evolución previa del electorado masculino (el voto de los hombres no permaneció estable respecto a 1931) como por el análisis de los estudios de caso, se puede defender que si solo hubiesen votado los hombres el giro conservador también se habría producido, ya que en los resultados electorales ni las mujeres ni los hombres fueron determinantes por separado. Esto se debe a que el comportamiento electoral de los españoles estaba guiado, más que por el sexo, por la posición socioeconómica, por la coyuntura del país y por las identidades personales.

Asimismo, los estudios de caso han mostrado que, sobre la base de las estadísticas disponibles, el que los partidos de izquierdas se presentasen a los comicios en candidaturas separadas, frente a las coaliciones de las derechas, fue lo que impidió a estas opciones políticas traducir sus apoyos en escaños de una forma más proporcional. Por supuesto, sobre esto a las electoras no se les puede atribuir ninguna responsabilidad.

Además, el que las mujeres, igual que los hombres, votasen en mayor medida a las derechas que a otras opciones ideológicas tiene que analizarse desde la perspectiva de que los signos de modernidad no son siempre los mismos. En este caso, su comportamiento electoral tiene de moderno que, en general, mostraron un grado relativamente alto de independencia a la hora de optar por sus opciones preferidas, lo cual no debe extrañar porque la mayoría de españolas tenía tanta (o tan poca) formación política como los hombres. De todas formas, para sustentar mejor estos planteamientos, que tienen ciertas limitaciones porque no existen en España datos electorales disgregados por sexo que permitan conocer con certeza cómo hubiesen sido los resultados de los comicios si solo hubiesen sufragado las mujeres, sería adecuado tener presente las tesis de los estudios históricos sobre aquellos países en los que sí se conoce el comportamiento electoral diferenciado por sexos en los comicios que inauguraron el sufragio 
universal en cada lugar. Por este motivo sería interesante trabajar en una perspectiva comparada entre España y esos países ${ }^{52}$.

En el caso de confirmarse a nivel nacional estas conclusiones provisionales y parciales sobre la inaugural experiencia electoral de las mujeres, sería fundamental profundizar en un futuro en el análisis sobre en qué medida se transformó el modelo de género hegemónico (y los subalternos en pugna) tras su obtención de los derechos políticos. También sería interesante el análisis de si esta experiencia fue un paso adelante en el proceso de su emancipación y empoderamiento y de si influyó en la modificación de las identidades culturales de las españolas.

\footnotetext{
$\overline{52}$ Por ejemplo: Maza, Erika, "Catolicismo, anticlericalismo y la extensión del sufragio a la mujer en Chile", en Estudios Públicos, 58 (1995), pp. 137-197; Valobra, Adriana María, "La ciudadanía política de las mujeres y las elecciones de 1951", en Anuario del Instituto de Historia Argentina, 8 (2008), pp. 71-74.
} 